\title{
Erratum: The promise of neuroprotective agents in Parkinson's disease
}

\author{
Stacey E. SeidI ${ }^{1}$ and Judith A. Potashkin ${ }^{2 *}$ \\ ${ }^{1}$ The Department of Biological Sciences, DePaul University, Chicago, IL, USA \\ 2 The Cellular and Molecular Pharmacology Department, The Chicago Medical School, RFUMS, North Chicago, IL, USA \\ ${ }^{*}$ Correspondence: judy.potashkin@rosalindfranklin.edu
}

Edited by:

Nick Andrews, Children's Hospital Boston, USA

Reviewed by:

Anders Haunso, Merck, UK

Zubair Ahmed, University of Birmingham, UK

\section{A commentary on}

The promise of neuroprotective agents in Parkinson's disease

by Seidl, S. E., and Potashkin, J. A., (2011).

Front. Neurol. 2:68. doi: 10.3389/fneur. 2011.00068

After our article was published online, it was brought to our attention that in Table 1 we incorrectly stated the findings from a study testing the safety of
Isradipine (Simuni et al., 2010). We have thus changed the text in the table to reflect the results that Isradipine has been shown to be safe and well-tolerated in PD patients.

\section{REFERENCES}

Simuni, T., Borushko, E., Avram, M. J., Miskevics, S. Martel, A., Zadikoff, C., et al. (2010). Tolerability of isradipine in early Parkinson's disease: a pilot dose escalation study. Mov. Disord. 25, 2863-2866.

Received: 27 March 2013; accepted: 15 April 2013; published online: 01 May 2013.
Citation: Seidl SE and Potashkin JA (2013) Erratum: The promise of neuroprotective agents in Parkinson's disease. Front. Neurosci. 7:69. doi: 10.3389/fnins. 2013.00069

This article was submitted to Frontiers in Neuropharmacology, a specialty of Frontiers in Neuroscience.

Copyright (c) 2013 Seidl and Potashkin. This is an open-access article distributed under the terms of the Creative Commons Attribution License, which permits use, distribution and reproduction in other forums, provided the original authors and source are credited and subject to any copyright notices concerning any thirdparty graphics etc. 
Table 1 | Neuroprotective Agents in PD models.

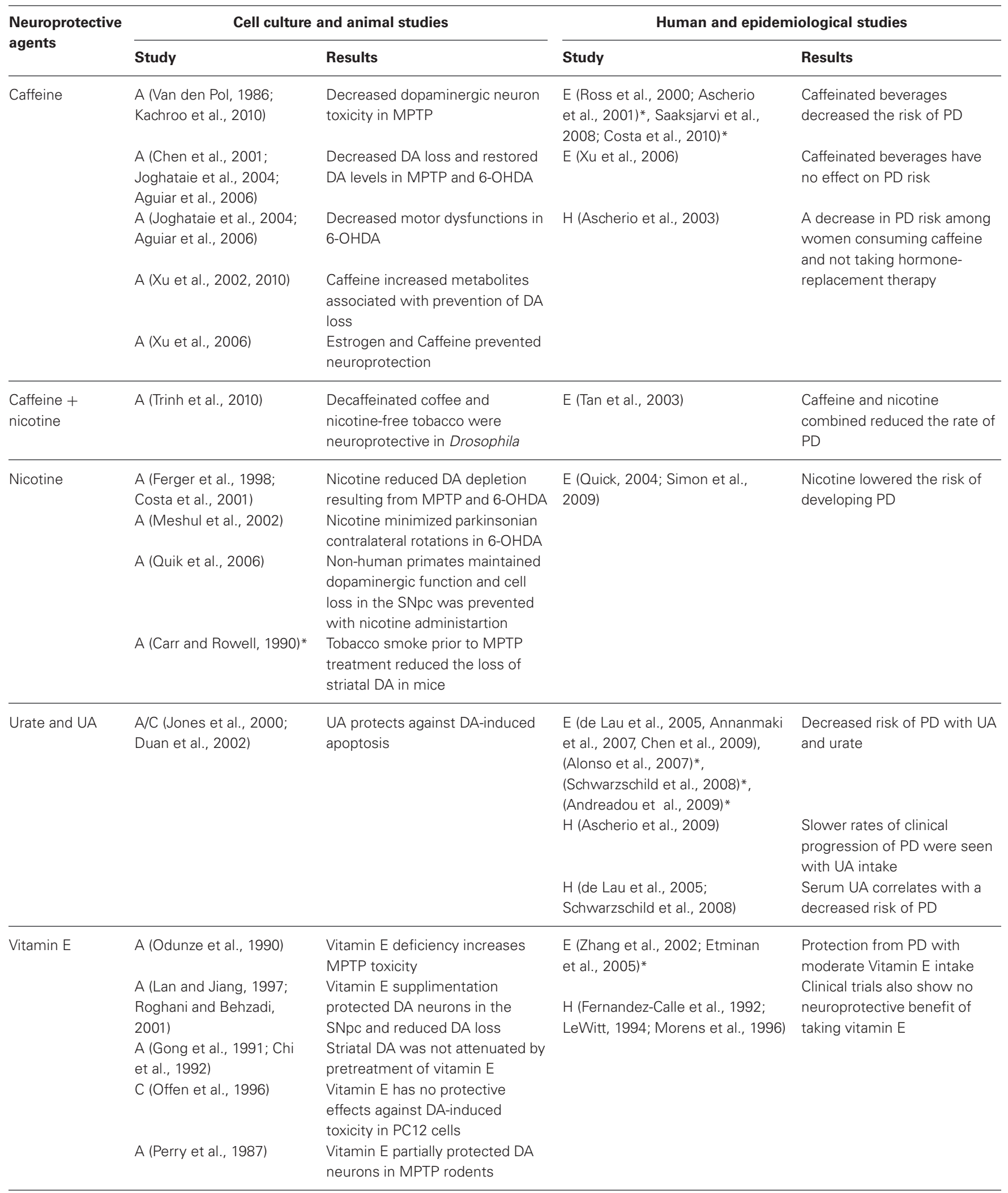




\section{Table 1 | Continued}

\begin{tabular}{|c|c|c|c|c|}
\hline $\begin{array}{l}\text { Neuroprotective } \\
\text { agents }\end{array}$ & \multicolumn{2}{|c|}{ Cell culture and animal studies } & \multicolumn{2}{|c|}{ Human and epidemiological studies } \\
\hline $\begin{array}{l}\text { Vitamin } E+ \\
\text { vitamin } C\end{array}$ & & & H (Fahn, 1992) & $\begin{array}{l}\text { Both vitamins combined } \\
\text { decreased PD progression in } \\
\text { early stage patients }\end{array}$ \\
\hline Vitamin C & & & $\begin{array}{l}\text { H (Scheider et al., 1997; Zhang } \\
\text { et al., 2002; Etminan et al., 2005) } \\
\text { H (Perlmutter, 1988) }\end{array}$ & $\begin{array}{l}\text { Few beneficial effects are } \\
\text { seen with vitamin } C \text { and even } \\
\text { an increased risk of PD } \\
\text { Vitamin } C \text { intake reduced the } \\
\text { risk of PD by } 40 \%\end{array}$ \\
\hline Vitamin D & $\begin{array}{l}\text { C (Butler et al., 2011) } \\
\text { H/A (Gash et al., 1996; } \\
\text { Kordower et al., 2000), } \\
\text { (Gill et al., 2003) } \\
\text { C/A (Wang et al., 2001; } \\
\text { Smith et al., 2006) } \\
\text { A (Holick, 2007) }\end{array}$ & $\begin{array}{l}\text { Disruption of Vitamin D's } \mathrm{Ca}^{2+} \\
\text { homeostasis properties } \\
\text { accelerated SNpc dopaminergic } \\
\text { neuron loss } \\
\text { GDNF stimulation by Vitamin D } \\
\text { can alleviated PD symptoms in } \\
\text { primates and PD patients } \\
\text { Vitamin D produced beneficial } \\
\text { effects against PD characteristics } \\
\text { Vitamin D increased } \\
\text { neuromuscular function in } \\
\text { parkinsonian rodents }\end{array}$ & E (Anderson et al., 1999) & $\begin{array}{l}\text { An increased risk of PD is } \\
\text { associated with high } \\
\text { consumption of vitamin D }\end{array}$ \\
\hline Beta-carotene & $\begin{array}{l}\text { (Perry et al., 1985, 1987; } \\
\text { Yong et al., 1986) }\end{array}$ & $\begin{array}{l}\text { Beta-carotene protected against } \\
\text { MPTP neurotoxicity in mice, but } \\
\text { not primates }\end{array}$ & E (Perlmutter, 1988) & $\begin{array}{l}\text { A decrease in the risk of PD } \\
\text { was seen with high } \\
\text { B-carotene intake }\end{array}$ \\
\hline Riboflavin & & & $\begin{array}{l}\text { E (Perlmutter, 1988; Murakami } \\
\text { et al., 2010) } \\
\text { E (Coimbra and Junqueira, 2003) }\end{array}$ & $\begin{array}{l}\text { Reduced risk of PD, with high } \\
\text { raboflavin intake by } 51 \% \\
\text { Riboflavin supplimentation } \\
\text { improved motor capacity of } \\
\text { PD patients }\end{array}$ \\
\hline $\begin{array}{l}\text { CoQ10 + } \\
\text { vitamin E }\end{array}$ & & & H (Shults et al., 2004) & $\begin{array}{l}\text { Combination showed } \\
\text { beneficial for PD patients, } \\
\text { however, Phase III trials } \\
\text { deemed it futile (NINDS, see } \\
\text { text footnote 1) }\end{array}$ \\
\hline Creatine & A (Matthews et al., 1999) & $\begin{array}{l}\text { Creatine protected against } \\
\text { MPTP-induced DA depletion in } \\
\text { the SNpc }\end{array}$ & $\begin{array}{l}\text { Investigators (2006) } \\
\text { Investigators (2008) }\end{array}$ & $\begin{array}{l}\text { Creatine delayed the } \\
\text { progression of PD by } 50 \% \\
\text { Creatine showed efficacy as a } \\
\text { neuroprotective agent in PD } \\
\text { and is currently in Phase III } \\
\text { trials (NET-PDLS, see text } \\
\text { footnote 2) }\end{array}$ \\
\hline $\begin{array}{l}\text { CoQ10 + } \\
\text { creatine }\end{array}$ & A/H (Yang et al., 2009) & $\begin{array}{l}\text { Combination showed a } \\
\text { neuroprotective effect in chronic } \\
\text { MPTP and humans }\end{array}$ & & \\
\hline
\end{tabular}




\section{Table 1 | Continued}

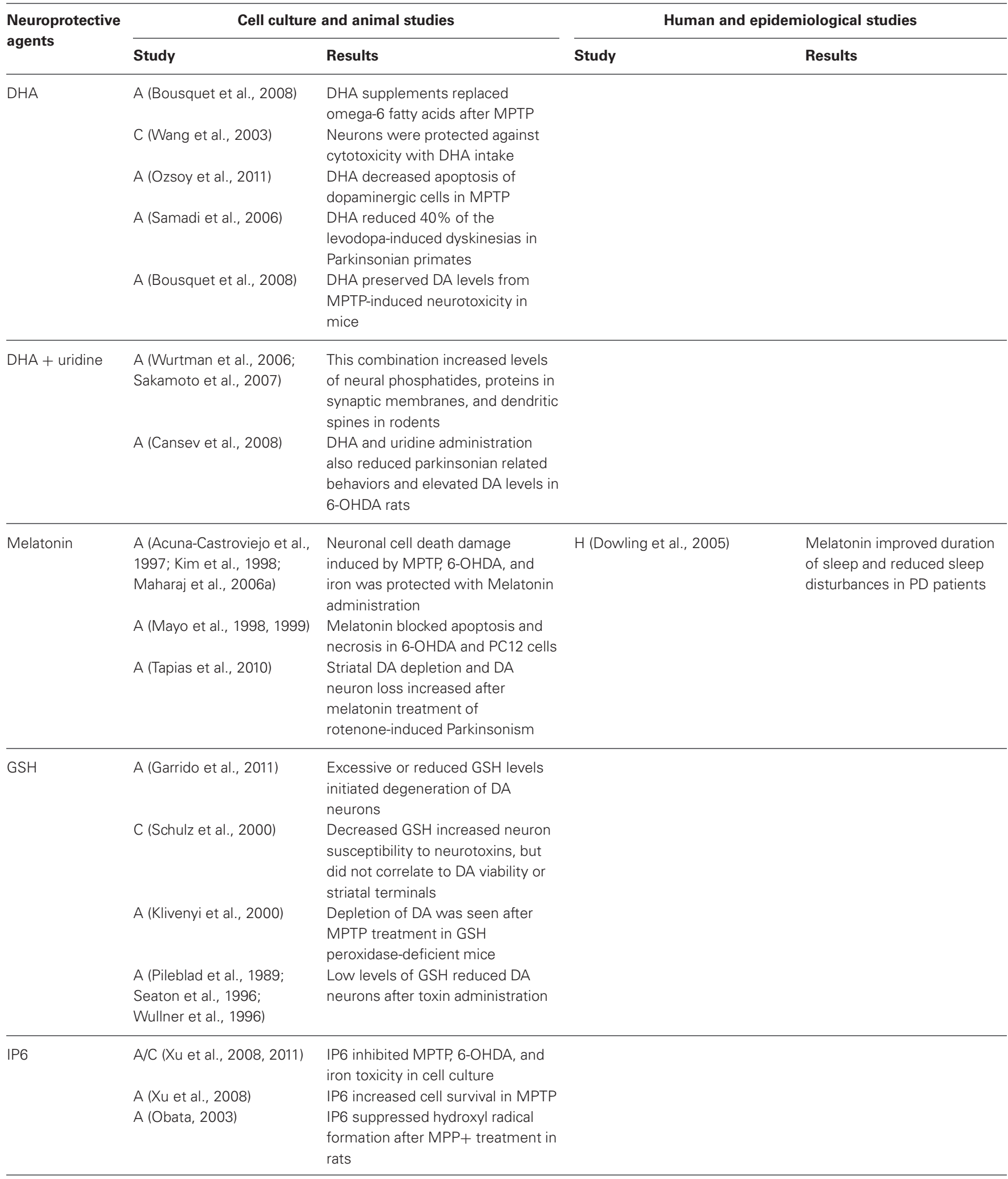


Table 1 | Continued

\begin{tabular}{|c|c|c|c|c|}
\hline \multirow{2}{*}{$\begin{array}{l}\text { Neuroprotective } \\
\text { agents }\end{array}$} & \multicolumn{2}{|c|}{ Cell culture and animal studies } & \multicolumn{2}{|c|}{ Human and epidemiological studies } \\
\hline & Study & Results & Study & Results \\
\hline \multirow[t]{4}{*}{$\begin{array}{l}\text { NSAID } \\
\text { (ibuprofen+ } \\
\text { aspirin) }\end{array}$} & $\begin{array}{l}\text { A/C (Bilodeau et al., 1995; } \\
\text { Kaufmann et al., 1997; } \\
\text { Aubin et al., 1998; Saini }\end{array}$ & $\begin{array}{l}\text { NSAID's protected against } \\
\text { neuronal death and dopaminergic } \\
\text { neurotoxicity }\end{array}$ & $\begin{array}{l}\text { E (Chen et al., 2003; Ton et al., } \\
\text { 2006; Wahner et al., 2007; Gao } \\
\text { et al., 2011) }\end{array}$ & NSAID's lowered the risk of PD \\
\hline & $\begin{array}{l}\text { et al., 1998; Casper et al., } \\
\text { 2000; Sairam et al., 2003) }\end{array}$ & & $\begin{array}{l}\text { E (Chen et al., 2005; Ton et al., } \\
\text { 2006; Driver et al. 2011, Gao } \\
\text { et al., 2011) }\end{array}$ & $\begin{array}{l}\text { A reduction in PD risk was } \\
\text { observed with Ibuprofen, but not } \\
\text { NSAIDS or Acetaminophen }\end{array}$ \\
\hline & A/C (Esposito et al., 2007) & $\begin{array}{l}\text { NSAIDS showed neuroprotection } \\
\text { in MPTP, 6-OHDA, and in vitro }\end{array}$ & E (Becker et al., 2011) & $\begin{array}{l}\text { NSAID's and aspirin showed no } \\
\text { association with altering the risk } \\
\text { of PD }\end{array}$ \\
\hline & $\begin{array}{l}\text { A (Casper et al., 2000; } \\
\text { Morioka et al., 2004; } \\
\text { Carrasco et al., 2005) }\end{array}$ & $\begin{array}{l}\text { Ibuprofen protected DA neurons } \\
\text { against glutamate toxicity and } \\
\text { decreased MPTP toxicity }\end{array}$ & $\begin{array}{l}\text { E (Bower et al., 2006; Hernan } \\
\text { et al., 2006) } \\
\text { E (Samii et al., 2009, Seroka, } \\
\text { 2010) }\end{array}$ & $\begin{array}{l}\text { Increased risk of PD shown with } \\
\text { moderate aspirin intake } \\
\text { lbuprofen reduced the risk of } \\
\text { developing PD in humans by } 40 \%\end{array}$ \\
\hline Isradipine & A (Ilijic et al., 2011) & $\begin{array}{l}\text { Isradipine showed neuroprotection } \\
\text { against 6-OHDA }\end{array}$ & H (Simuni et al., 2010) & $\begin{array}{l}\text { Isradipine has been shown to be } \\
\text { safe and well-tolerated in PD } \\
\text { patients }\end{array}$ \\
\hline Phenylbutyrate & $\begin{array}{l}\text { C (Gardian et al., 2004; } \\
\text { Zhou et al., 2011) } \\
\text { A (Zhou et al., 2011) }\end{array}$ & $\begin{array}{l}\text { Phenylbutyrate protected DA } \\
\text { neurons in the SNpc } \\
\text { Reduced deterioration in motor } \\
\text { and cognitive function in mice }\end{array}$ & & \\
\hline \multirow[t]{2}{*}{ Ex-4 } & A (Li et al., 2009) & $\begin{array}{l}\text { Protected DA neuron } \\
\text { degeneration, preserved DA levels, } \\
\text { and improved motor function in } \\
\text { rodents }\end{array}$ & & \\
\hline & $\begin{array}{l}\text { A/C (Harkavyi et al., 2008; } \\
\text { Li et al., 2009) }\end{array}$ & $\begin{array}{l}\text { Ex-4 protected ventral } \\
\text { mesencephalic dopaminergic cells } \\
\text { in culture, reverse nigral lesions, } \\
\text { and protected against 6-OHDA } \\
\text { toxicity }\end{array}$ & & \\
\hline \multirow[t]{4}{*}{ Rasagiline } & $\begin{array}{l}\text { C (Heikkila et al., 1985; } \\
\text { Huang et al., 1999; Speiser } \\
\text { et al., 1999; Maruyama } \\
\text { et al., 2000; Sagi et al., } \\
\text { 2001; Youdim et al., 2001a) }\end{array}$ & $\begin{array}{l}\text { Reduces MPTP and 6-OHDA } \\
\text { toxicity in PC12 and SH-SY5Y cells }\end{array}$ & $\begin{array}{l}\text { H (Hauser et al., 2009; Olanow } \\
\text { et al., 2009) }\end{array}$ & $\begin{array}{l}\text { Rasagiline reduced the long-term } \\
\text { progression and symptoms in PD }\end{array}$ \\
\hline & $\begin{array}{l}\text { A (Kupsch et al., 2001, } \\
\text { Sagi et al., 2001). }\end{array}$ & $\begin{array}{l}\text { Rasagiline prevented nigrostriatal } \\
\text { damage induced by MPTP in } \\
\text { primates }\end{array}$ & & \\
\hline & A (Blandini et al., 2004) & $\begin{array}{l}\text { Rasagiline increased DA neuron } \\
\text { survival in lesioned SNpc and } \\
\text { improved motor impairments }\end{array}$ & H (Rascol et al., 2011) & $\begin{array}{l}\text { Rasagiline in a Phase III delayed } \\
\text { the need for antiparkinsonian } \\
\text { drugs and patients had lower } \\
\text { scores on the Parkinson's disease } \\
\text { rating scale }\end{array}$ \\
\hline & C (Murer et al., 2001) & $\begin{array}{l}\text { Rasagiline increased expression of } \\
\text { neurotrophins }\end{array}$ & & \\
\hline Minocycline & A/C (Du et al., 2001) & $\begin{array}{l}\text { Minocycline blocked } \\
\text { MPTP-induced degeneration of DA } \\
\text { neurons in the SNpc preventing } \\
\text { loss of striatal DA and its } \\
\text { metabolites. Minocycline } \\
\text { treatment also inhibited MPP+ } \\
\text { mediated inducible NO synthase } \\
\text { expression in vivo and blocked } \\
\text { NO-induced neurotoxicity in vitro }\end{array}$ & H (Investigators, 2006) & $\begin{array}{l}\text { Minocycline was deemed } \\
\text { effective in Phase II slowing the } \\
\text { progression of PD in patients. } \\
\text { An 18-month follow up study } \\
\text { showed no safety concerns with } \\
\text { its use (Investigators, 2008), } \\
\text { leading to Phase III trials }\end{array}$ \\
\hline
\end{tabular}


Table 1 | Continued

\begin{tabular}{|c|c|c|c|c|}
\hline $\begin{array}{l}\text { Neuroprotective } \\
\text { agents }\end{array}$ & \multicolumn{2}{|c|}{ Cell culture and animal studies } & \multicolumn{2}{|c|}{ Human and epidemiological studies } \\
\hline & $\begin{array}{l}\text { A (Faust et al., 2009; } \\
\text { Radad et al., 2010) }\end{array}$ & $\begin{array}{l}\text { DA neuroprotection by Minocycline } \\
\text { was seen in a Drosophila model of } \\
\text { PD and after rotenone toxicity in } \\
\text { rodents }\end{array}$ & & \\
\hline & A/C (Yang et al., 2003) & $\begin{array}{l}\text { Minocycline exacerbated MPTP } \\
\text { damage to DA neurons in vitro and } \\
\text { in vivo }\end{array}$ & & \\
\hline & A (Diguet et al., 2004) & $\begin{array}{l}\text { Minocycline treatment in primates } \\
\text { and mice produced more } \\
\text { severe/rapid parkinsonism, } \\
\text { behavior deficits, and greater loss } \\
\text { of nerve endings }\end{array}$ & & \\
\hline $\begin{array}{l}\text { Minocycline }+ \\
\text { creatine }\end{array}$ & & & H (NET-PD, 2006) & $\begin{array}{l}\text { Reduced progression in PD } \\
\text { patients in Phase II }\end{array}$ \\
\hline
\end{tabular}

C, cell culture; A, animal; H, human; and E, epidemiological. Studies showing gender specificity, where males show favorable results are denoted (*). 